\title{
Coronal Holes in Global Complexes of Activity
}

\author{
Vladimir N. Obridko and Bertha D. Shelting \\ IZMIRAN, Kaluzhskoe Shosse 4, Troitsk, Moscow 142190, Russia \\ Correspondence should be addressed to Vladimir N. Obridko; obridko@izmiran.ru
}

Received 17 April 2015; Accepted 28 June 2015

Academic Editor: Alberto J. Castro-Tirado

Copyright (C) 2015 V. N. Obridko and B. D. Shelting. This is an open access article distributed under the Creative Commons Attribution License, which permits unrestricted use, distribution, and reproduction in any medium, provided the original work is properly cited.

\begin{abstract}
We propose a new concept that considers the global complexes of activity as a combination of global and local fields. Traditionally, the complexes of activity have been identified from observations of active regions (ARs). Here, we show that a complex of activity comprises both (AR) and coronal holes (CHs). Our analysis is based on observations of magnetic fields of various scales, SOHO/MDI data, and UV observations of $\mathrm{CH}$. The analysis has corroborated the existence of complexes of activity that involve $\mathrm{AR}$ and equatorial $\mathrm{CH}$. Both $\mathrm{AR}$ and $\mathrm{CH}$ are embedded in an extended magnetic region dominated by the magnetic field of one sign, but not strictly unipolar. It is shown that the evolution of $\mathrm{CH}$ and $\mathrm{AR}$ is a single process. The relationship between the fields of various scales in the course of a cycle is discussed.
\end{abstract}

\section{Introduction}

The study of complexes of solar activity has quite a long history. It began, most likely, with the work by Gnevyshev [1], who proved that besides the 11-year cycles of solar activity there were shorter periods when the level of activity increased. He called them "pulses of activity."

These results were presented later by Eigenson et al. [2].

The following step was associated with Mt. Wilson magnetographic measurements covering the entire surface of the Sun. It was then that a more adequate term, "complexes of solar activity," was proposed in [3-5] to characterize longterm (from months to years) enhancements of solar activity in a certain range of heliolongitudes. These authors actually claimed that the complexes of activity must comprise not only $\mathrm{AR}$, but also unipolar magnetic regions and, hence, $\mathrm{CH}$, which suggested their global nature.

In [6], Obridko and Shelting introduced the notion of a "global complex of activity," combining both local and global field structures.

The study of such a complex feature requires the use of large volumes of data, including characteristics of local and global fields and information on processes in the photosphere, chromosphere, corona, and even heliospheric and geomagnetic disturbances, covering a long time interval.
This is one of the reasons why the notion of the complex of activity has lately fallen into disuse. At present, the authors often prefer to consider instead the "complexes of active regions" (or even the "complexes of sunspot groups"). These do not require the use of extensive databases and, most importantly, are not supposed to live long. Many authors (e.g., see [7-11]) consider the complex of active regions as two or more ARs connected by a common magnetic field, whose components or parts interact in the course of evolution.

The analysis of global complexes of activity is additionally complicated by the need to take into account the interactions of fields differing in their both spatial scales and intensity. Large-scale fields are, obviously, connected with the global magnetic field [12], and their evolution is controlled by processes deep under the photosphere, probably, at the bottom of the convection zone. On the other hand, the structure of a complex must depend strongly on the evolution of intense fields of AR, which are shallow features (5-10 Mm deep).

As a rule, large-scale fields are associated with open field regions (OFRs), that is, the field whose force lines extend into interplanetary space and are carried away by the solar wind. These are the regions where $\mathrm{CHs}$ arise. Wang et al. [13] identified $\mathrm{CH}$ with OFR. Ever since, these terms have been used interchangeably to refer to $\mathrm{CH}$ observed in UV and X-rays on the disk and at the limb. Of course, this is 
a purely statistical coincidence. The open field regions are calculated in a complex way within the concept of the sourcesurface potential field with a lot of additional assumptions. The occurrence of $\mathrm{CH}$ depends not only on the magnetic structure, but also on the relative contribution of various heating mechanisms, which may somewhat differ in different $\mathrm{CH}$.

If the relationship between OFR and $\mathrm{CH}$ is quite understandable and the equal use of both terms is physically justified, another analogy that is frequently drawn is not as evident. Some authors identify large quasi-unipolar regions (UR) with open field regions and define $\mathrm{CH}$ boundaries simply as the boundaries of UR. Such an approach is physically groundless. Large quasi-unipolar regions may be the remnants of AR. Then, their field is shallow, and the field lines close inside the same region. Such regions do not form OFR, and their unipolarity index differs much from unity [6].

In the course of evolution, the OFR, $\mathrm{CH}$, and $\mathrm{AR}$ form a single complex. Its properties are as follows. Magnetic fields in the chromosphere and corona that depart from the radial direction by less than $20^{\circ}$ are the sites of occurrence of $\mathrm{CH}$ [14]. Despite the physical identity of these objects, their boundaries may not fully coincide and $\mathrm{CH}$ may occur somewhat later than OFR.

Two or three rotations after the occurrence of a $\mathrm{CH}$, its shape and size begin to change affected by the AR located between the conjugate $\mathrm{CH}$. As a result, a saddle-type feature appears in the transversal field in the photosphere close to the $\mathrm{CH}$ boundaries.

Outside the photometric $\mathrm{CH}$, the magnetic vectors converge to the conjugate active regions to form a single complex of activity. This effect is pronounced best of all in large AR. A relationship between large $\mathrm{AR}$ and $\mathrm{CH}$ was noted earlier $[15,16]$ in spite of the well-known fact that the source of the large-scale magnetic field lies deep at the bottom of the convection zone [17], while sunspots and AR occur much closer to the surface.

The lifetime of $\mathrm{CH}$ ranging from 2 to 3 rotations to 1.5 years, exceeding the lifetime of the associated AR. A decrease or disappearance of AR leads to corresponding changes in $\mathrm{CH}$.

Obridko and Shelting [6] analyzed two typical time intervals in Cycle 23: from October 1999 to March 2001 (Carrington rotations 1954-1974) and from December 2002 to December 2003 (Carrington rotations 1997-2010). The former covers the entire period of maximum of Cycle 23 and the latter covers the period of reversal of the polar field. The second period is characterized by a specific, stable structure of the quasi-equatorial dipole, which is often observed at the decline of the cycle.

These intervals differ significantly by the structure of the large-scale field. In the first interval, the field structure is not completely stable. Its typical variation time is $2-3$ rotations. In the second one, a stable two-sector structure is maintained throughout 14 rotations. Such a structure can be described by equatorial dipole with the axis in the vicinity of the equator.

Comparison of the positions of $\mathrm{CH}$ and $\mathrm{AR}$ on the disk involves certain difficulties, because it is not clear $a$ priori what we must take for the boundary of a $\mathrm{CH}$ even if observed at one and the same wavelength. Therefore, in the first part of our work, we compare the positions of AR with the position of the open magnetic field region (OFR) inside a complex (Section 2.) Then, in Section 3, we analyze the relative positions of OFR and AR. The analysis in Section 4 includes $\mathrm{CH}$, for which purpose we discuss again the problem of $\mathrm{CH}$ photometric characteristics (Sections 4.1 and 4.2) and consider the mutual position of $\mathrm{CH}, \mathrm{AR}$, and magnetic fields.

\section{Comparative Analysis of Evolution of the OFR Total Area and the Number of Sunspot Groups in a Complex}

To begin with, we have analyzed the evolution of the OFR area and the number of sunspot groups. The analysis is based on OFR calculations. The position and structure of OFR were determined from WSO photospheric data (http://wso.stanford.edu/) by the standard method described in our earlier work $[18,19]$. First, we calculated the Legendre polynomial coefficients by the least square method under potential approximation and, then, all magnetic field components in a spherical layer from the photosphere to the source surface. Calculations were fulfilled for the source surface at $2.5 R_{\odot}$ from the center of the Sun. The first ten harmonics of the expansion were taken into account. This method is quite similar to the method applied by Hoeksema [20,21]. Then, the field lines were traced from the nodes of the uniform grid on the source surface, where they are open by definition, down to their intersection with the chosen spherical surface.

The shape and positions of OFR on the synoptic map for each rotation determined in such a way were, then, compared with SOHO/MDI data on AR and magnetic fields in the photosphere (http://soi.stanford.edu/magnetic/index5.html and http://soi.stanford.edu/magnetic/index6.html). The area and position of each AR were taken at the peak of its evolution.

The number of field lines emerging from the grid with a uniform distribution of nodes $(72 \times 30)$ on the source surface and concentrated in a given region of open magnetic field can serve as a measure of the area of this OFR. The number of ARs in a complex increases with the increase of the OFR area. Although the correlation coefficient is not high $(0.39 \pm 0.13$ in the first case and $0.47 \pm 0.15$ in the second one), the positive trend is evident. This positive correlation corroborates a genetic link between OFR and sunspot groups at the early stage of evolution. One or two rotations after an OFR is formed, a $\mathrm{CH}$ arises over it and, somewhat later, a sunspot group appears at its periphery.

\section{Relative Positions of OFR and AR}

As shown in [14], a CH arises in the open field region, where the field lines are mainly radial. While moving away from the region of strictly radial field, the filed lines in the ambient photosphere become more and more inclined. Up to the inclination of $20^{\circ}$, the $\mathrm{CH}$ still exists, but its contrast decreases gradually creating a kind of " $\mathrm{CH}$ penumbra" until, at the inclination of $50^{\circ}$, it merges with undisturbed photosphere. The region, in which the inclination of field lines is more than 
TABLE 1

\begin{tabular}{lcc}
\hline & All AR & AR in the vicinity to OFR \\
\hline Sum of all AR areas & 198324 & $147733 / 198334=0.745$ \\
Sum of AR areas $>600 \mathrm{msh}$ & $93096 / 198334=0.469$ & $76756 / 93096=0.824$ \\
Sum of AR areas $<600 \mathrm{msh}$ & $105238 / 198238=0.531$ & $70977 / 105238=0.674$ \\
Total number of ARs & 470 & $324 / 470=0.689$ \\
Number of ARs with areas $>600 \mathrm{msh}$ & 89 & $73 / 89=0.820$ \\
Number of ARs with areas $<600 \mathrm{msh}$ & 381 & $251 / 381=0.659$ \\
\hline
\end{tabular}

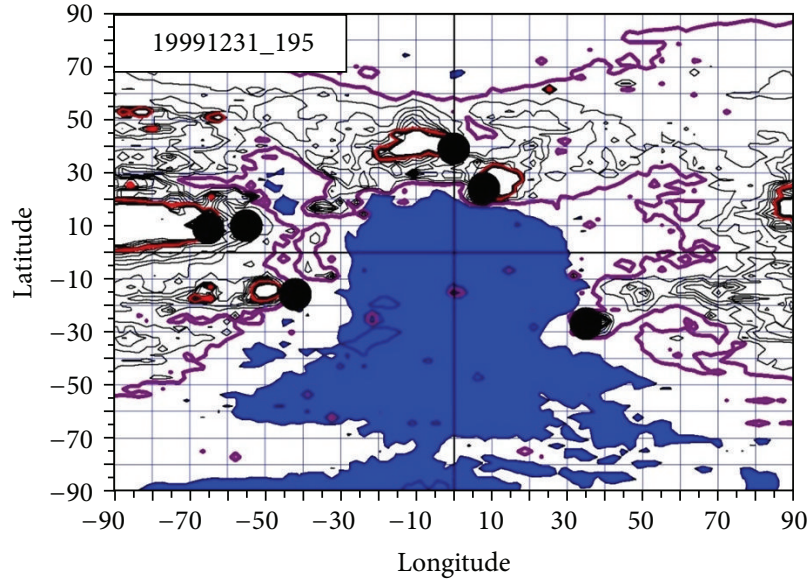

(a)

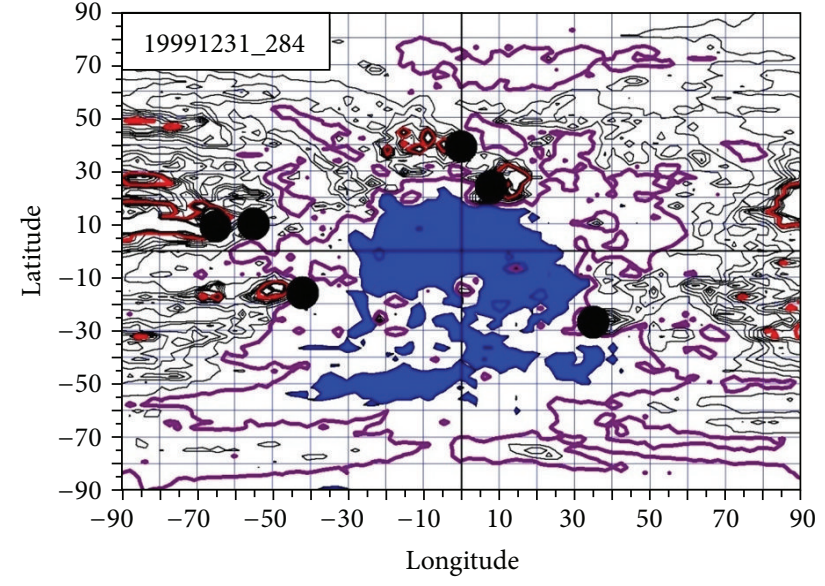

(b)

FIGURE 1: Heliographic maps of the Sun image in the lines $195 \mathrm{~A}$ (a) and $284 \mathrm{~A}$ (b). The figure shows contour lines of equal brightness, including the lines of double mean brightness (magenta) and tenfold mean brightness (red). The area shaded blue is the region where the brightness is lower than the average on the given day. The black circles are sunspot groups.

$20^{\circ}$ but less than $50^{\circ}$, is precisely where the $\mathrm{AR}$ conjugates with $\mathrm{CH}$ arising.

To quantitatively estimate the vicinity of sunspot groups to OFR, we have analyzed the entire set of data. In doing so, we assumed that a sunspot group is located close to $\mathrm{CH}$ (or, in our case, OFR) if its center lies at less than 10 heliographic degrees from the $\mathrm{CH}$ boundary.

For the period under consideration, 470 ARs were observed, whose total area was $198324 \mathrm{msh}$. We analyzed the number of sunspot groups and their total area depending on their proximity to $\mathrm{CH}$ boundaries. It turned out that 324 sunspot groups (i.e., 68.9\%) appeared in the vicinity of $\mathrm{CH}$ boundaries. And although there were only 89 groups with the area at the peak of evolution $>600 \mathrm{msh}$, the absolute majority of them $(82 \%)$ were located close to $\mathrm{CH}$ boundaries. The same is true for the index of the total sunspot area. The area of sunspot groups located in the vicinity of OFR was $74.5 \%$ of the total area. Note that the main contribution (82.4\%) to the total area of sunspot groups in the vicinity of $\mathrm{CH}$ is made by the $>600$ msh groups. Thus, both large and small sunspot groups are observed in the vicinity of $\mathrm{CH}$, but the relative number of large ones is greater (see Table 1).

\section{Relative Positions of Photometric $\mathrm{CH}$ and $\mathrm{AR}$}

We analyzed $\mathrm{CH}$ images in two wavelengths $195 \mathrm{~A}$ and $284 \mathrm{~A}$ taken on the day when the complexes under consideration were at the center of the visible disk. Each image was corrected for projection and, then, represented in the form of a rectangular heliographic map. Two ov such maps are presented in Figure 1. The figure shows contour lines of equal brightness, including the lines of double mean brightness (magenta) and tenfold mean brightness (red). The area shaded blue is the region where the brightness was lower than the average on the given day. The black circles are sunspot groups.

In Figure 1, the area shaded blue is the coronal hole, which we identify as the region where the brightness is lower than the average on the given day. One can see that transition from $\mathrm{CH}$ to $\mathrm{AR}$ systems of photometric contour lines occurs between the lines of mean and double mean brightness. In this case, the accuracy of the boundary does not really matter. Despite the general similarity, the coronal holes observed in the lines mentioned above differ essentially. On the image taken in the $284 \mathrm{~A}$ line, the $\mathrm{CH}$ is somewhat smaller and its boundary is much more indented than on the $195 \mathrm{~A}$ image obtained on the same day.

4.1. Comparison of the Minimum, Mean, and Maximum $\mathrm{CH}$ Brightness in Two Spectral Lines. Since the $\mathrm{CH}$ photometric boundary is referenced to the mean brightness of the disk on a given day, it can be determined, in principle, by sunspot activity. In [14], we analyzed data for a long time interval and showed that the mean disk brightness in the $284 \mathrm{~A}$ line 

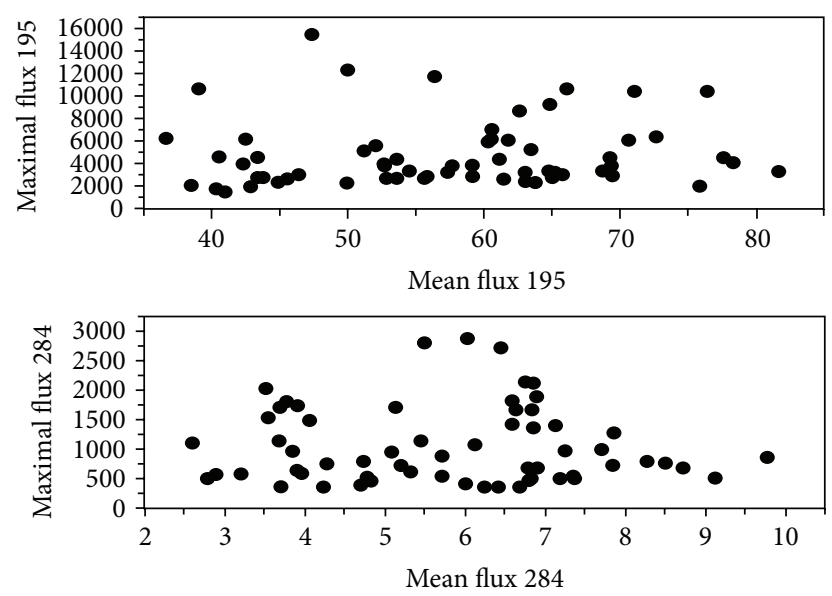

FIGURE 2: Comparison of the maximum and mean brightness values.

depends on the phase of the cycle, but it is not determined by sunspot activity.

In the database used in this work, we have found the minimum, maximum, and mean brightness on each image.

In Figure 2, we compare the maximum and mean brightness values in both wavelengths for 64 days of $\mathrm{CH}$ observation. If AR contributed significantly to the calculated mean disk brightness, we could expect a noticeable relation between the mean and maximum brightness. Figure 2 shows that such a relation is lacking.

Even more representative is Figure 3, which compares all three calculated brightness values. There is a fairly good correlation (0.758) between the minimum brightness values in both wavelengths. It should be borne in mind that these are direct observational data without any additional calculations. We just find a single pixel in which the brightness is minimal.

The correlation between the mean brightness values is clearly pronounced, the correlation coefficient amounting to 0.951. If we remove two points standing apart (days when solar flares occurred), the correlation coefficient will increase to 0.982 . The maximum brightness values are virtually uncorrelated. The red line is plotted taking into account all points, and the correlation coefficient is 0.56 . The blue line plotted without the two flare-related points gives the correlation coefficient of 0.27 .

4.2. The Problem of Photometric Boundaries. Since our task is to study the AR position with respect to $\mathrm{CH}$, the problem of correctly marking the boundaries of the latter arises again. The $\mathrm{CH}$ boundaries are often defined by the visual contrast without estimating the boundary brightness numerically. We might introduce a standard value for the ratio between the minimum $\mathrm{CH}$ brightness and the brightness at the boundary. Unfortunately, this method sometimes yields uncertain result, because, unlike the mean brightness, the minimum brightness is determined with a significant error (see Figure 3). We also used standard values for the "umbra" and "penumbra" in fractions of the mean disk brightness on a given day. These methods are valid for the statistical study of $\mathrm{CH}$ occurrence rate and relation to high-speed streams (see [14] and references therein). However, it is inadequate for comparing the positions of individual $\mathrm{CH}$ and AR. In the previous sections where we compared OFR and $\mathrm{AR}$, the boundaries were determined automatically from the footpoints of the field lines. Studying $\mathrm{CH}$ boundaries requires an additional numerical value to be introduced.

To find the photometric boundaries, we have assumed that the area $S$ within the closed isophotes IP normalized to the mean brightness will increase with increasing isophote value. The growth rate of the area must also increase gradually reaching its maximum as the isophote equals the $\mathrm{CH}$ boundary value. With further increase in the isophote value (i.e., in the quasiuniform, undisturbed medium), the increase of the area is expected to slow down. Thus, inside a $\mathrm{CH}$, the area will increase by a certain quantity $\Delta S$ with each isophote step. The increase is supposed to be maximal at the $\mathrm{CH}$ boundary.

This procedure was applied to all $\mathrm{CH}$ (the summary picture is shown in Figure 4).

Figure 4 illustrates the increase in the relative area $\Delta S$ with increasing isophote value IP. IP is normalized to the mean brightness on a given day, and $S$ is normalized to the total disk area on the same day corrected for projection. A step $\Delta I P$ equals 0.025 . The relative area is seen to reach its maximum at 0.725 for $195 \mathrm{~A}$ and at 1.075 for $284 \mathrm{~A}$. A slight bend of the curve for $284 \mathrm{~A}$ at IP $=0.2-0.4$ is likely to be associated with the darkest part ("umbra") of $\mathrm{CH}$. This means that the umbra boundary is at IP $=0.2-0.3$ and the outer boundary of the penumbra is at $\sim 1.0$.

Figure 5 represents a CH observed on 11 July 1999 at the wavelengths of $195 \mathrm{~A}$ (Figure 5(a)) and $284 \mathrm{~A}$ (Figure 5(b)). On both panels, one can see the umbra of $\mathrm{CH}$.

The values of the photometric boundaries found above (1.075 for $284 \mathrm{~A}$ and 0.725 for $195 \mathrm{~A}$ ) were used to calculate the areas and internal mean brightness of $64 \mathrm{CH}$. The results are compared in Figure 6. It should be remembered that the values of the internal mean brightness are normalized to the mean brightness of the disk at the selected wavelength on the day of observation. One can see a pronounced negative correlation between the area and brightness. In larger $\mathrm{CH}$, the contrast is higher (as it should be). The relative brightness 

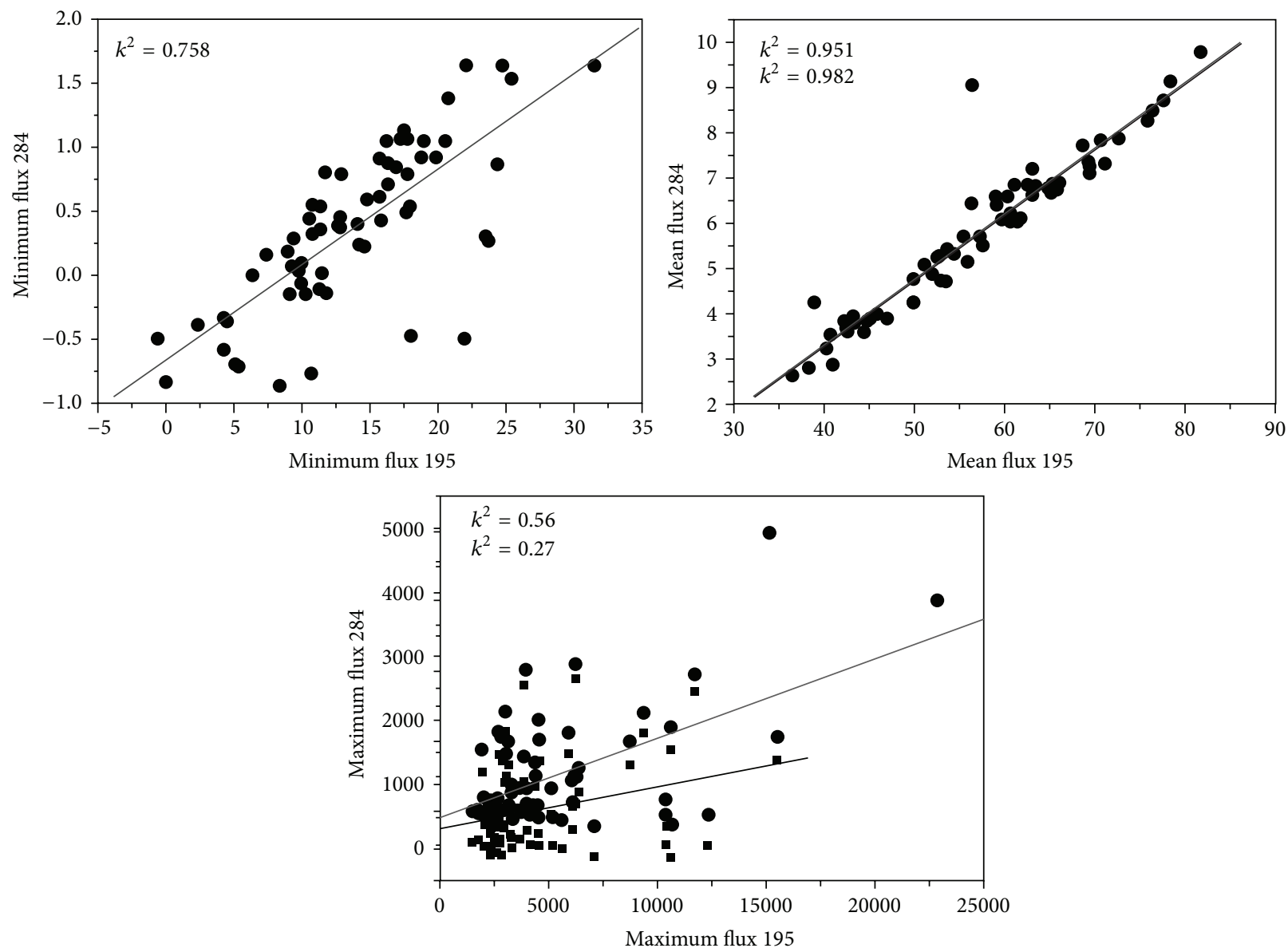

Figure 3: Comparison of the calculated minimum, mean, and maximum brightness values in two lines.

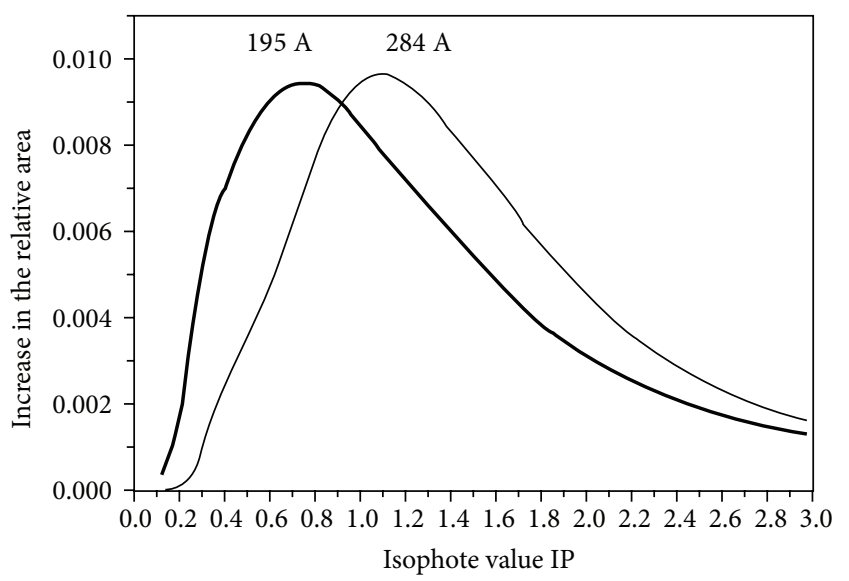

FIGURE 4: The increase in the relative area $\Delta S$ with increasing isophote value IP.

value in $284 \mathrm{~A}$ is somewhat larger (contrast lower) than in $195 \mathrm{~A}$. The areas of reasonably large $\mathrm{CH}$ within the selected boundaries virtually coincide at both wavelengths.

4.3. Comparison with the Location of Sunspot Groups. In Section 3, we compared the areas and location of AR with respect to OFR. Since the open field regions were identified from synoptic maps for one rotation, the AR data were also taken once, at the peak of their evolution, and all sunspot groups were included independent of their lifetime. In our further consideration, we are using the images of $64 \mathrm{CHs}$ corresponding to OFR at the time of their passage through the central meridian. The coordinates and areas of AR are taken for the same day. The data are converted into a Cartesian line grid, a sample of which is shown in Figure 1.

The concentration of sunspot groups in the vicinity of $\mathrm{CH}$ (see Table 2) is approximately the same as in the case of OFR. In the second period, it is somewhat higher, probably, because the structure of the large-scale magnetic field was then more stable.

The number of AR decreases gradually as the distance from $\mathrm{CH}$ increases (see Table 2 and Figure 7). At 10 heliographic degrees, their number decreases by a factor of 1.5-2.0. This effect is particularly noticeable for large sunspot groups (>100 msh).

4.4. Magnetic Field Structure in the Vicinity of $\mathrm{CH}$. As shown earlier by Obridko and Shelting [14], the direction of the magnetic field in the vicinity of $\mathrm{CH}$ changes rapidly. At the center of $\mathrm{CH}$ (in the umbra), the field departs slightly from the normal. The angle between the field lines and the normal 


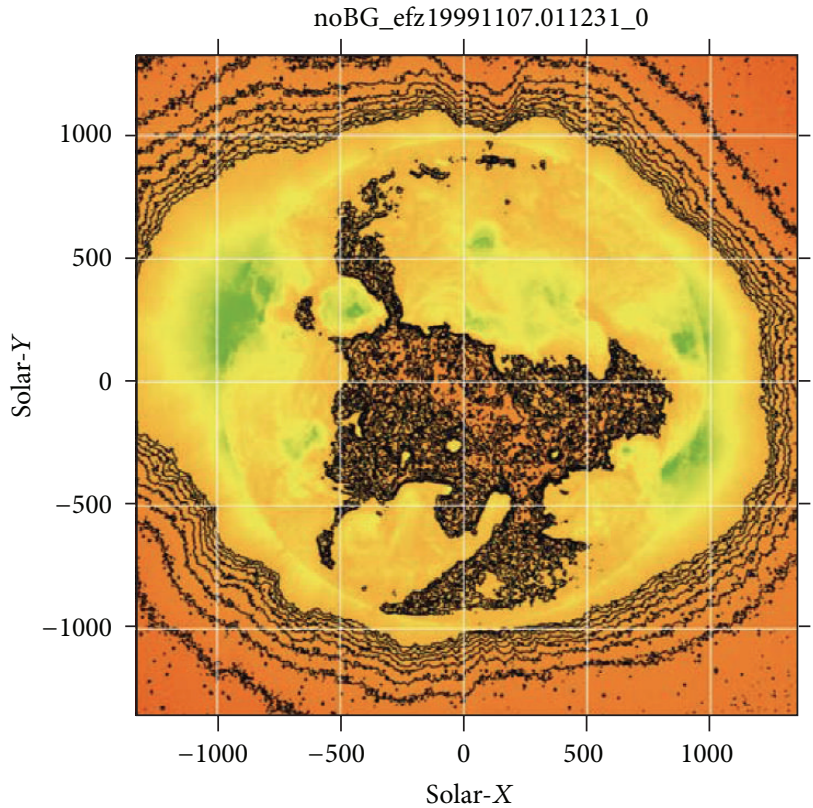

(a)

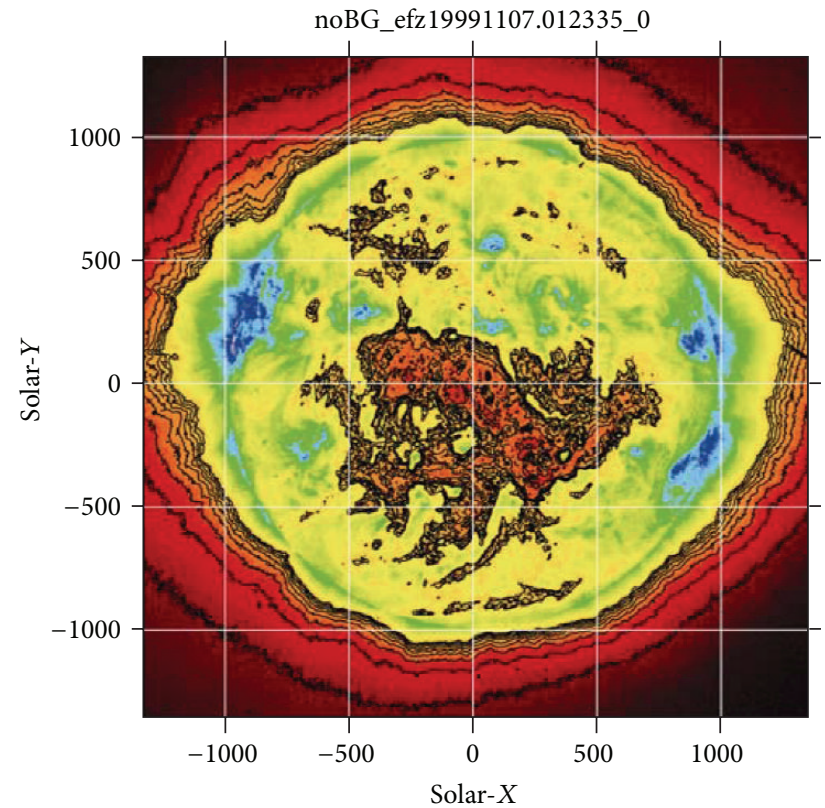

(b)

Figure 5: CH observed on 11 July 1999 at the wavelengths of 195 A (a) and 284 A (b).
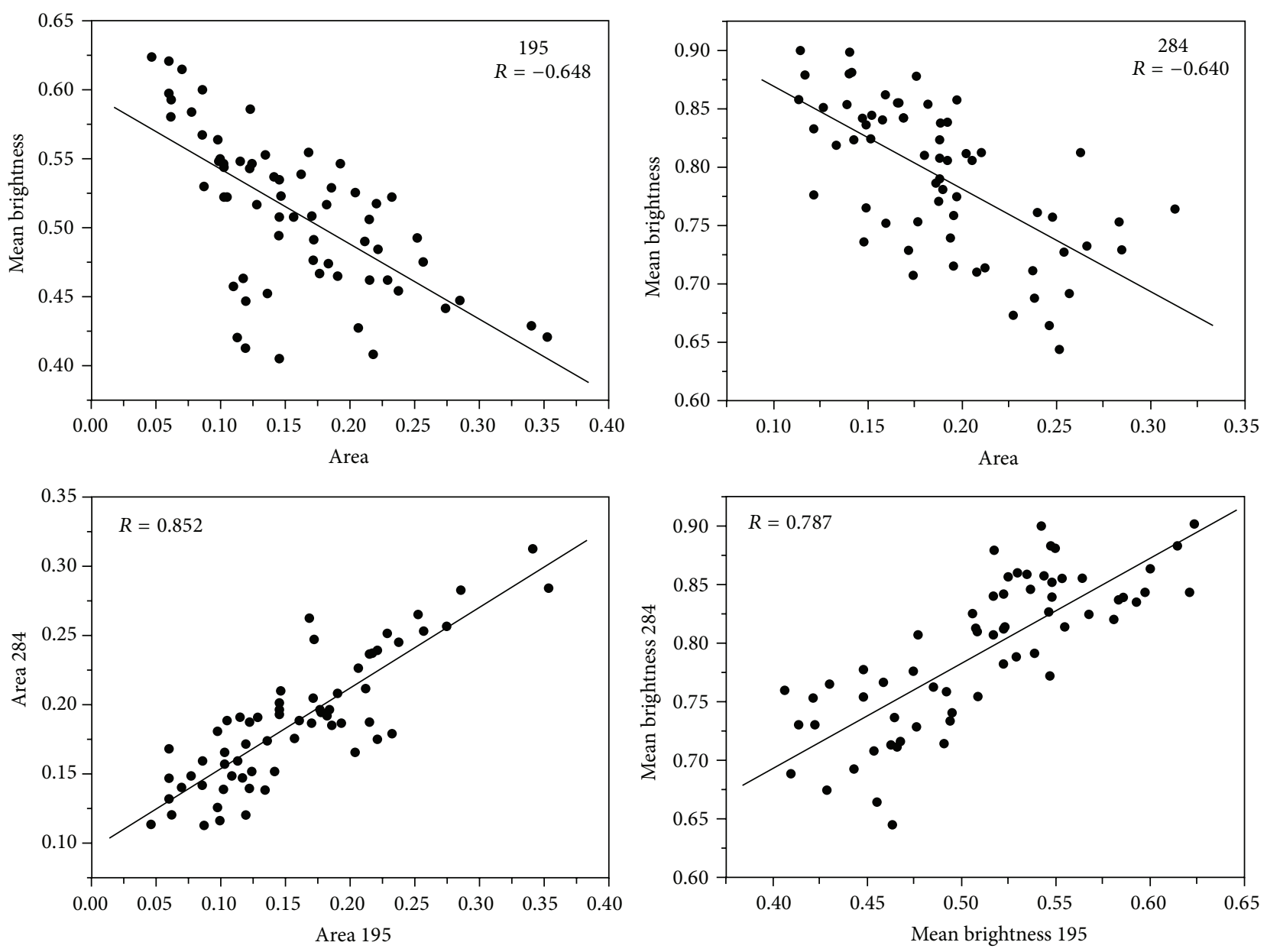

Figure 6: Comparison of $\mathrm{CH}$ areas and brightness in two lines. 


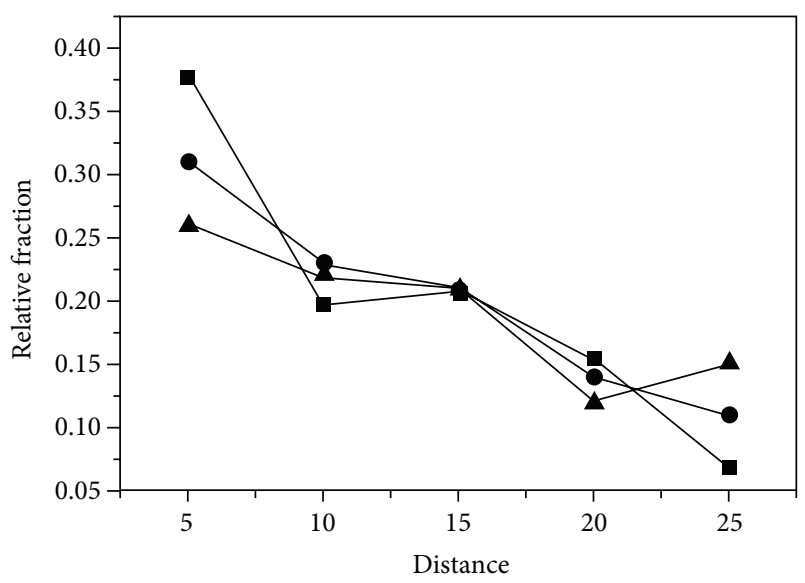

FIGURE 7: The relative fraction of AR of different areas as a function of distance from $\mathrm{CH}$ (circles are all $\mathrm{AR}$, squares are groups of areas more than $100 \mathrm{msh}$, and triangles are groups of areas less than $100 \mathrm{msh})$.

TABLE 2

\begin{tabular}{lcccc}
\hline & \multicolumn{2}{c}{$\begin{array}{c}\text { First period: } \\
\text { rotations 1954-1974 } \\
\text { (Sept. 1999-Dec. 2001) }\end{array}$} & $\begin{array}{c}\text { Second period: } \\
\text { rotations 1997-2010 } \\
\text { (Nov. 2002-Dec. 2003) }\end{array}$ \\
\hline & All AR & $\begin{array}{c}\text { AR near } \\
\text { CH }\end{array}$ & All AR & $\begin{array}{c}\text { AR near } \\
\mathrm{CH}\end{array}$ \\
Total area & 41804 & $\begin{array}{c}24462 \\
(63.3 \%)\end{array}$ & 21034 & $\begin{array}{c}17049 \\
(81.1 \%)\end{array}$ \\
$\begin{array}{l}\text { Total number } \\
\text { of ARs }\end{array}$ & 284 & $\begin{array}{c}186 \\
(65.5 \%)\end{array}$ & 147 & $111(75.5 \%)$ \\
\hline
\end{tabular}

to the surface does not exceed $20^{\circ}$. As the distance from $\mathrm{CH}$ increases (in the penumbra), the field lines begin to diverge rapidly, and their angle with the normal at $\mathrm{CH}$ boundary reaches $50^{\circ}$. The calculations were performed with the use of the classical PSFF model with all inherent restrictions [20]. Figure 8 illustrates the $\mathrm{CH}$ observed at the center of the disk on 18 April 2000. Figure 8(a) represents an original SOHO image in the instrumental reference frame without processing and without regard to projection distortions with only some contrasting performed. One can readily see a coronal hole at the center of the disk. Figures 8(b) and 8(c) were plotted in the Cartesian coordinates with the longitude and latitude values corrected for projection. The blue areas on Figure $8(\mathrm{~b})$ correspond to the brightness lower than the mean value. Figure 8 (c) shows the structure of the tangential magnetic field calculated from the PSFF model [20]. The black contours on Figure 8(c) outline the regions where the brightness is lower than the mean value. The size of the arrows is proportional to the strength of the field. It is evident that the $\mathrm{CH}$ is located exactly at the point from which the lines come.

The dependence of angle $\gamma$ between the magnetic field vector and the normal to the solar surface on the distance to the $\mathrm{CH}$ photometric boundaries is shown in Figure 9. One can see that this angle increases up to $50^{\circ}$ already at a distance of $7-10^{\circ}$.
4.5. Relationship between the Number of Sunspot Groups in the Vicinity of $\mathrm{CH}$ and Its Area. If we compare the number of ARs with $\mathrm{CH}$ area within the zone of $\pm 40^{\circ}$ from the visible disk center, the result will differ from what is observed when comparing the number of ARs with OFR (see Section 2 above). The situation is rather uncertain, unlike the previous case when we had a weakly pronounced upward trend in the number of ARs with increasing OFR area.

Two complexes of activity were analyzed in rotations 1954-1974.

In the first one (18 points, 16 degrees of freedom), the correlation coefficient is $0.086 \pm 0.081$. The Student's $T$ criterion for the angular coefficient is 0.34549 ; that is, the correlation is absent completely.

In the second complex (18 points, 16 degrees of freedom), the correlation coefficient is $0.599 \pm 0.452$. The Student's Tcriterion for the angular coefficient is 2.989 ; that is, we have a low negative correlation, but the trend is clearly pronounced with the reliability $>99 \%$.

Two more complexes were studied in rotations 1997-2010.

In the third complex, (14 points, 12 degrees of freedom) the correlation coefficient is $0.025 \pm 0.023$. The Student's $T$-criterion for the angular coefficient is 0.345 ; that is, the correlation is absent completely.

In the fourth complex (13 points, 12 degrees of freedom), the correlation coefficient is $0.549 \pm 0.425$. The Student's $T$ criterion for the angular coefficient is 3.831; that is, we have a negative correlation with a low, not too reliable correlation coefficient; however, the trend is clearly pronounced with the reliability $>99 \%$.

In general, one can see weak negative correlation.

On the one hand, this may be due to different criteria in selecting sunspot groups. However, it is most probably the result of different generation mechanisms of OFR and $\mathrm{CH}$. The open field regions are determined completely by the deep magnetic field, while the occurrence of $\mathrm{CH}$ is largely controlled by the heating mechanisms. Thus, sunspot groups are not passively located in the vicinity of $\mathrm{CH}$ but affect the brightness and photometric structure of the latter owing to the lateral heating. AR arise mainly in the vicinity of OFR and $\mathrm{CH}$, but they decrease the $\mathrm{CH}$ area and shift its boundaries by heating.

\section{Conclusion}

Thus, we have shown that open field regions and $\mathrm{CH}$ form the basis of a global complex of activity. A complex of activity is not a random cluster of AR characterized by a common position on the disk and similar evolution during several solar rotations. It appears that the complexes of activity are a necessary part in the general scheme of largescale organization of solar activity on both spatial and long temporal scales. Their formation and evolution are controlled not only by the surface local fields, but also by the global processes.

The mechanisms responsible for the solar activity on a global scale are the large-scale solar dynamo and meridional flows. 


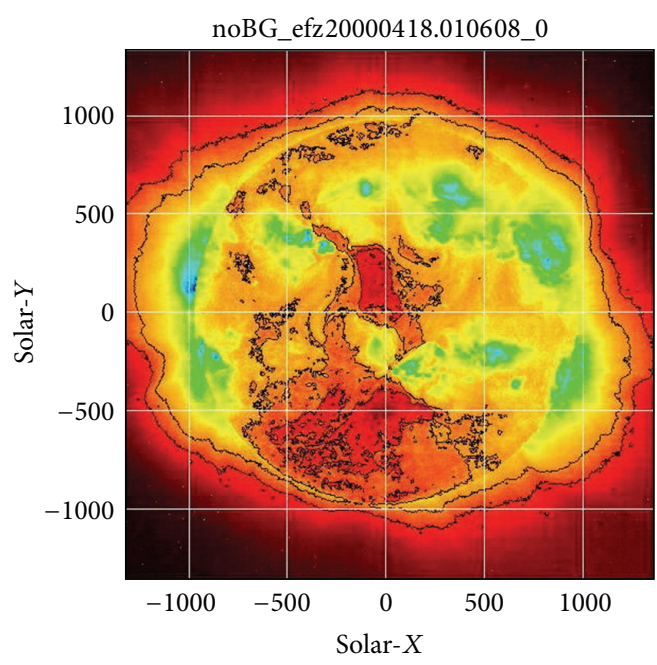

(a)

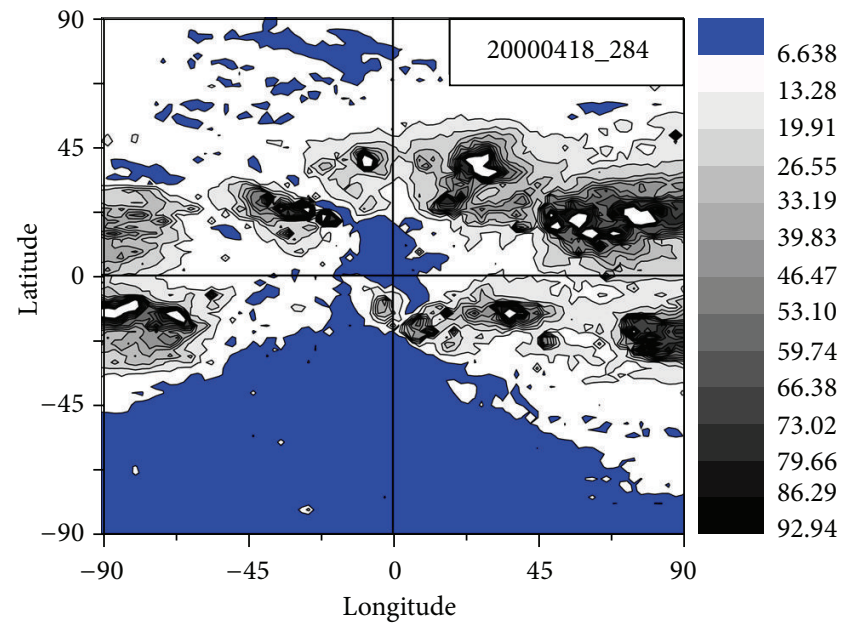

(b)

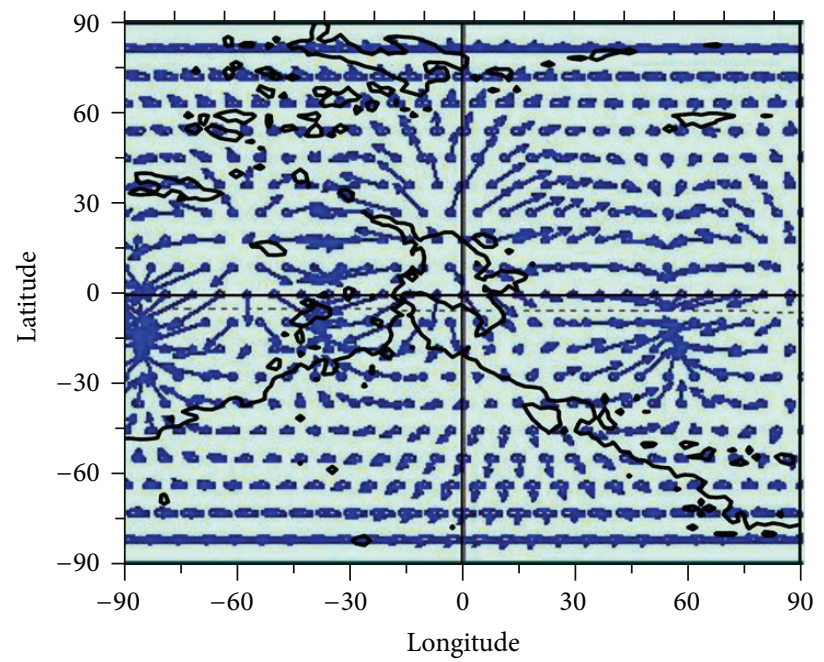

(c)

Figure 8: CH observed at the center of the disk on 18 April 2000. Panel (c) shows the structure of the tangential magnetic field.

Today it is clear that the second condition for the occurrence of $\mathrm{CH}$ at midlatitudes, apart from the open configuration of the global magnetic field, is the presence of AR with strong local field in their neighborhood. The relationship between $\mathrm{CH}$ and $\mathrm{AR}$ was revealed in earlier work based on Skylab data [13, 22, 23]. The appearance of the open field of $\mathrm{CH}$ is usually accompanied by the formation of two systems of closed field lines. Later it was shown $[24,25]$ that, sometimes, the change of boundaries and energy balance in $\mathrm{CH}$ is largely determined sporadic and pulse flows of hot plasmas (X-ray "jets") and regular flows of energy from the surrounding AR. AR in the vicinity of $\mathrm{CH}$ may be coupled with the latter dynamically or energetically.

Magnetic field is generated at the base of the convection zone as a result of interaction of the toroidal magnetic field and differential rotation ( $\Omega$-dynamo). The generated field emerges from the tachocline in the equatorial zone presumably in the form of individual tubes and appears at the surface as AR. In the process, a feature often arises in the vertical field, which looks darker than the surroundings in Xrays and $\mathrm{UV}$, that is, a $\mathrm{CH}$. At its periphery where the field lines depart from the radial direction, there appears AR. The physical mechanism responsible for concentration of active regions in the vicinity of $\mathrm{CH}$ is still unknown. Perhaps, it is due to different role of the deep and subsurface dynamo. In the open magnetic configuration, the vertical field associated with deep layers suppresses the subsurface mechanism of filed intensification. In the regions where the vertical field departs from the normal and its connection with deep layers weakens, the surface field is intensified, and an active region is formed. Thus, the two types of dynamo interact at the periphery of OFR. Besides, the coronal condensation over AR may, in turn, distort the photometric contour of $\mathrm{CH}$. Note that the AR rotation somewhat differs from that of the deep field. The surface fields rotate at Carrington velocity. The rotation of 


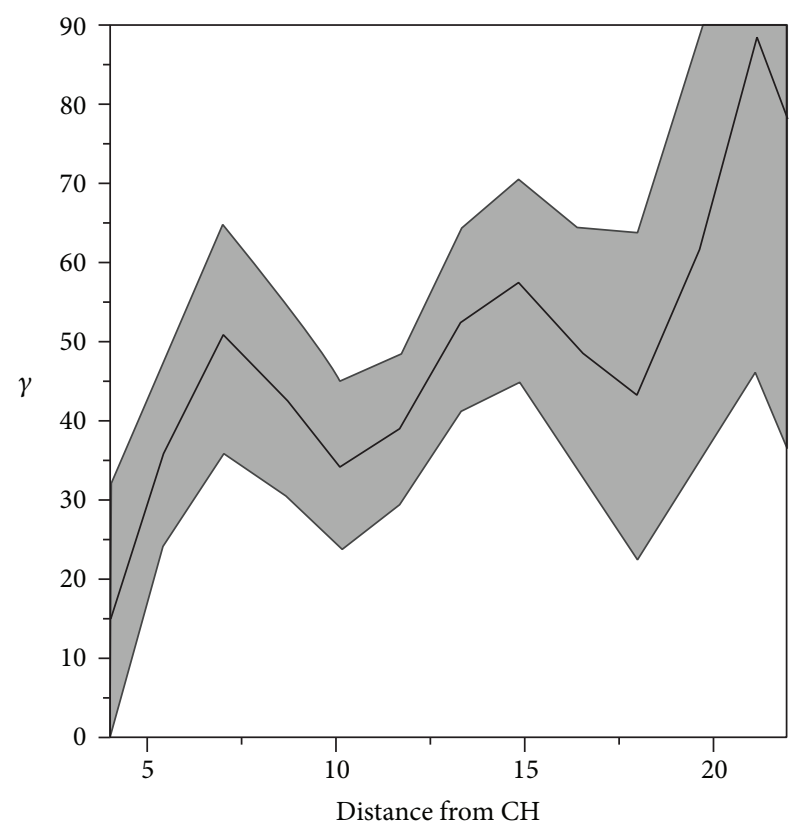

FIGURE 9: Angle $\gamma$ as a function of distance from the $\mathrm{CH}$ photometric boundary. The grey area shows the $95 \%$ confidence interval.

the deep field is more similar to the solid-body rotation and has a period of 27 days.

After a few rotations, the ARs usually decay. Their influence on the deep-field tube ceases gradually, and the structure of open field lines is restored. A new $\mathrm{CH}$ appears. However, it is not formed of the remnants of AR but is connected with the deep field.

\section{Conflict of Interests}

The authors declare that there is no conflict of interests regarding the publication of this paper.

\section{References}

[1] M. N. Gnevyshev, "On the nature of solar activity," Izvestiia Glavnoi astronomicheskoi observatorii v Pulkove, vol. 16, pp. 3645, 1938.

[2] M. S. Eigenson, M. N. Gnevyshev, A. I. Ol', and B. M. Rubashev, Solar Activity and Its Terrestrial Manifestations, Gostekhizdat, Moscow, Russia, 1948 (Russian).

[3] H. W. Babcock and H. D. Babcock, "The sun's magnetic field, 1952-1954," The Astrophysical Journal, vol. 121, p. 349, 1955.

[4] V. Bumba and R. Howard, "A study of the development of active regions on the sun," The Astrophysical Journal, vol. 141, article 1492, 1965.

[5] V. Bumba and R. Howard, "Large-scale distribution of solar magnetic fields," The Astrophysical Journal, vol. 141, p. 1502, 1965.

[6] V. N. Obridko and B. D. Shelting, "Global complexes of activity," Astronomy Reports, vol. 57, no. 10, pp. 786-796, 2013.

[7] V. N. Ishkov and E. I. Mogilevsky, Solar Activity, vol. 3, AlmaAta, Almaty, Kazakh Soviet Socialist Republic, 1983.
[8] E. I. Mogilevsky and N. S. Shilova, "Complexes of active regions on the sun," Izvestiya Krymskoi Astrofiz-Icheskoi Observatorii, vol. 94, p. 29, 1998.

[9] S. A. Yazev, "Coronal holes and complexes of activity," Notices of Irkutsk State University, Series Earth Sciences, vol. 3, no. 2, p. 226, 2010.

[10] S. A. Yazev, "Activity complexes on the sun during the 23rd solar cycle," Geomagnetism and Aeronomy, vol. 51, no. 7, pp. 879-887, 2011.

[11] S. A. Yazev, "NATO Advanced Study Institute. Advances in solar research at eclipses from ground and from space," Romanian Astronomical Journal, vol. 9, supplement 1, p. 87, 1999.

[12] B. P. Filippov, Y. V. Platov, A. Ajabshirizadeh, and D. V. Klepikov, "Polar coronal structures and the global magnetic field evolution through the cycle," Solar Physics, vol. 224, no. 1-2, pp. 277-284, 2004.

[13] Y.-M. Wang, S. H. Hawley, and N. R. Sheeley Jr., "The magnetic nature of coronal holes," Science, vol. 271, no. 5248, pp. 464-469, 1996.

[14] V. N. Obridko and B. D. Shelting, "Relationship between the parameters of coronal holes and high-speed solar wind streams over an activity cycle," Solar Physics, vol. 270, no. 1, pp. 297-310, 2011.

[15] P. McIntosh, "The solar cycle," in Proceedings of the 12th Summer Workshop on National Solar Observatory/Sacramento Peak, vol. 27 of ASP Conference Series, p. 14, ASP, San Francisco, Calif, USA, 1992.

[16] V. Bumba, M. Klvǎna, and J. Sýkora, "Coronal holes and their relation to the background and local magnetic fields," Astronomy \& Astrophysics, vol. 298, p. 923, 1995.

[17] E. E. DeLuca and P. A. Gilman, "The solar dynamo," in Solar Interior and Atmosphere, (A92-36201 14-92), pp. 275-303, University of Arizona Press, Tucson, Ariz, USA, 1991.

[18] V. N. Obridko and B. D. Shelting, "Structure of the heliospheric current sheet derived for the interval 1915-1916," Solar Physics, vol. 184, no. 1, pp. 187-200, 1999.

[19] V. N. Obridko and B. D. Shelting, "Structure and cyclic variations of open magnetic fields in the sun," Solar Physics, vol. 187, no. 1, pp. 185-205, 1999.

[20] J. T. Hoeksema and P. H. Scherrer, Solar Magnetic Field-1976 through 1985, WCDA, Boulder, Colo, USA, 1986.

[21] J. T. Hoeksema, Solar Magnetic Field-1985 through 1990, WCDA, Boulder, Colo, USA, 1991.

[22] R. H. Levine, "Large scale solar magnetic fields and coronal holes," in CHs and High Speed Wind Streams, J. B. Zirker, Ed., p. 103, Colorado Associated University Press, Boulder, Colo, USA, 1977.

[23] R. H. Levine, M. D. Altschuler, J. W. Harvey, and B. V. Jackson, "Open magnetic structures on the sun," Astrophysical Journal, vol. 215, p. 636, 1977.

[24] K. Shibata, N. Yokoyama, and M. Shimojo, "Coronal X-ray jets," in New Look at the Sun with Emphasis on Advanced Observations of Coronal Dynamics and Flares, S. Enome and T. Hirayama, Eds., p. 75, Nobeyama Radio Observatory, Nagano, Japan, 1994.

[25] E. I. Mogilevsky, "Energetics of coronal holes and recurrent geomagnetic disturbances," Geomagnetizm I Aeronomiya, vol. 35, no. 6, p. 11, 1995. 

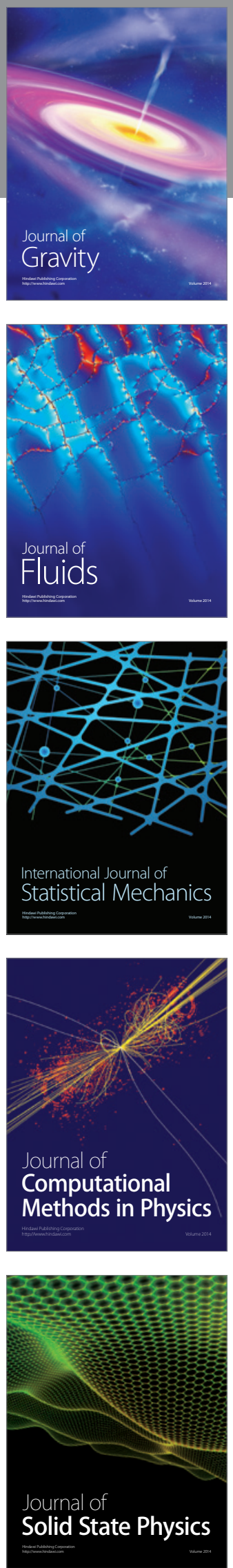

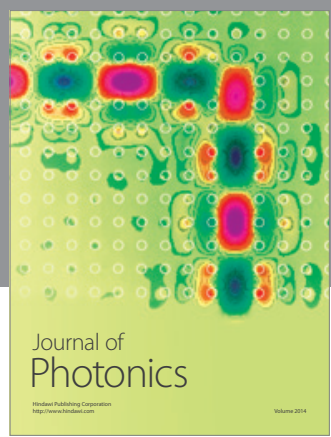

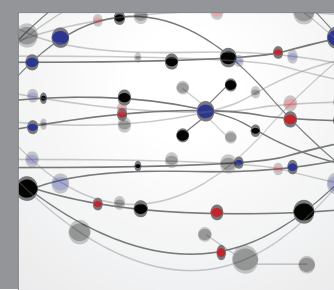

The Scientific World Journal

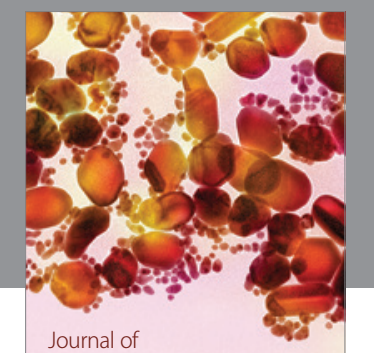

Soft Matter
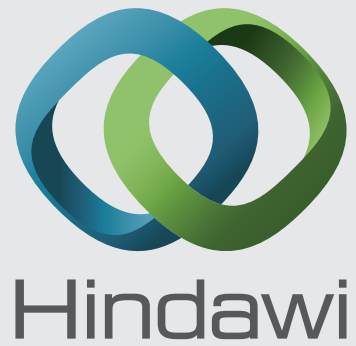

Submit your manuscripts at

http://www.hindawi.com
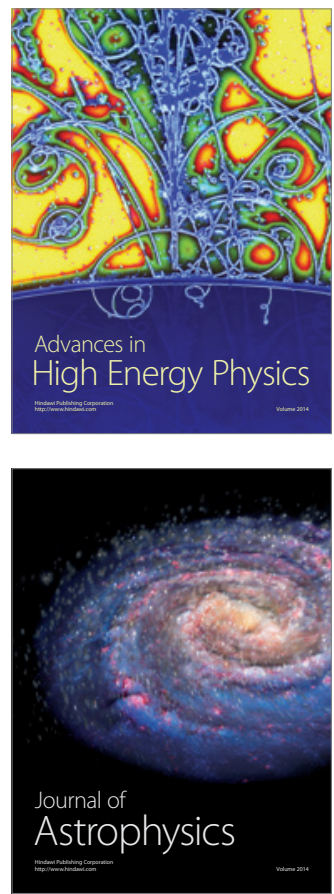
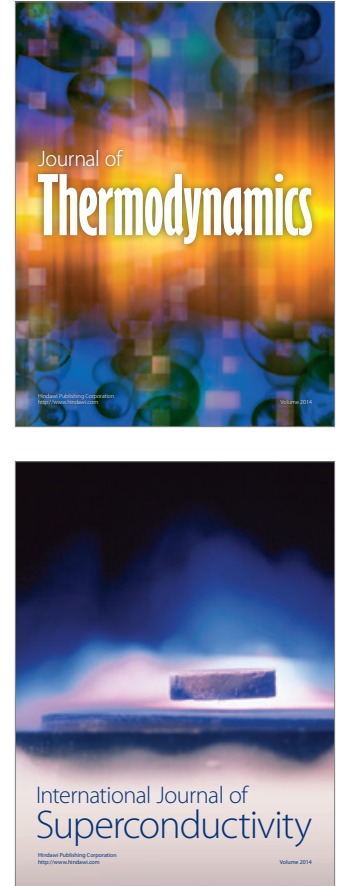
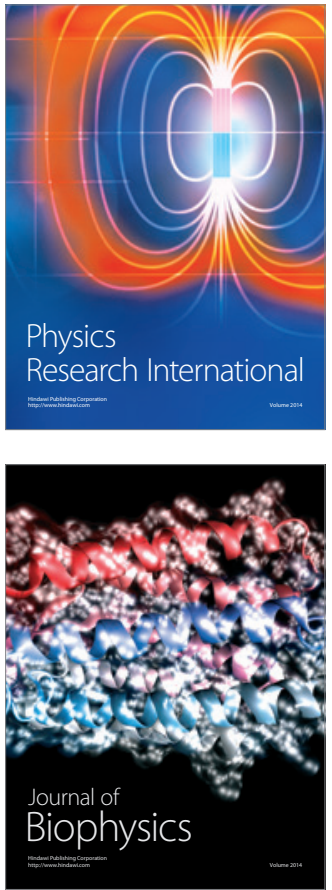
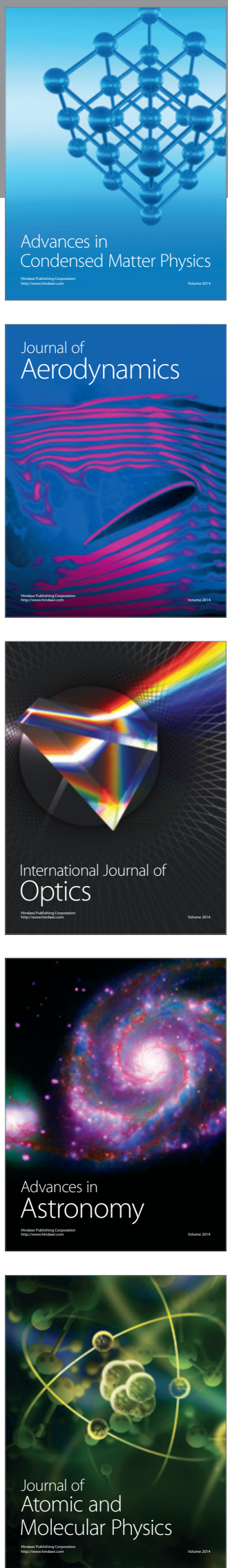